\title{
Biodegradation of Functionalized Nanocellulose
}

Benjamin P. Frank ${ }^{1}$, Casey Smith ${ }^{1}$, Emily R. Caudill ${ }^{2}$, Ronald S. Lankone ${ }^{1}$, Katrina Carlin ${ }^{1}$, Sarah J. Benware, ${ }^{2}$ Joel A. Pedersen ${ }^{2}$, D. Howard Fairbrother ${ }^{1}$

${ }^{1}$ Department of Chemistry, Johns Hopkins University, 3400 N Charles Street, Baltimore, MD 21218, United States

${ }^{2}$ Department of Chemistry, University of Wisconsin-Madison, 1101 University Avenue, Madison, Wisconsin 53706, United States

Supporting Information

23 Pages, 15 Figure, 9 Tables 


\section{Materials Characterization}

ATR-FTIR. Attenuated total reflectance Fourier-transform infrared spectroscopy data of CNF powders were obtained with a Nicolet iS5 spectrometer and an iD5 ATR attachment with a diamond stage using a scan range from $4000 \mathrm{~cm}^{-1}$ to $525 \mathrm{~cm}^{-1}$ at $0.964 \mathrm{~cm}^{-1}$ resolution with 32 scans collected and averaged per measurement.

CHN Elemental Analysis. CHN elemental analyses were performed to determine $\mathrm{DS}_{\text {overall }}$ for functionalized CNFs. Tests were performed on an Exeter Analytical CE440 CHN Analyzer at the Microanalysis Laboratory at University of Illinois at Urbana-Champaign. Dried samples (2.0 $3.0 \mathrm{mg}$ ) were weighed into consumable tin capsules, placed in an autosampler wheel, and purged with helium. The capsule was driven into a high temperature $\left(1000^{\circ} \mathrm{C}\right)$ furnace and combusted in pure oxygen under static conditions. The resulting combustion products containing carbon dioxide $\left(\mathrm{CO}_{2}\right)$, water $\left(\mathrm{H}_{2} \mathrm{O}\right)$, nitrogen $\left(\mathrm{N}_{2}\right)$ and oxides of nitrogen $\left(\mathrm{NO}_{\mathrm{x}}\right)$ were passed over copper to scrub excess oxygen and reduce oxides of nitrogen to elemental nitrogen. After scrubbing, the gases entered a mixing volume chamber to ensure a homogeneous mixture at constant temperature and pressure. The mixture then passed through a series of high-precision thermal conductivity detectors to determine the $\mathrm{CHN}$ content in two replicates of each sample.

Solid-State NMR. Solid-state NMR spectra were collected at the University of Wisconsin - Madison on a Bruker Avance III $500 \mathrm{MHz}$ spectrometer equipped with a 4 mm cross-polarization magic angle spinning (CP-MAS) probe. Cross polarization experiments were performed, wherein the peak intensity and area depend on both the abundance of the observed nuclei and the number of protons on nearby probed carbon nuclei (within $3-4 \AA$ ). The ${ }^{13} \mathrm{C}$ spectra were externally referenced using adamantine (upfield peak was set to $38.5 \mathrm{ppm}$ ). Spectra were acquired at $298 \mathrm{~K}$ at a rotational speed of $12 \mathrm{kHz}$. Other experimental acquisition details are previously reported. ${ }^{1}$ 
All spectra were processed in MestReNova, including baseline correction and manual phase correction. Peaks corresponding to amorphous or crystalline forms of CNF were assigned based on known chemical shifts, ${ }^{2}$ and the proportion of crystalline and amorphous character was determined by averaging these respective peak areas.

Variable contact time (VCT) cross polarization-magic-angle spinning (CP-MAS) experiments were conducted to estimate $\mathrm{DS}_{\text {overall }}$ from ${ }^{13} \mathrm{C}$ NMR data for two samples: phenyl ester CNF and DA-CNF-2. The molecular motion of the aromatic ring in the phenyl ester was expected to exhibit slower molecular motion than the long aliphatic chain in the dodecyl ester and lead to a shorter relaxation time constant of protons in the rotating frame $\left(T_{1 \rho \mathrm{H}}\right)$. Phenyl ester CNF and DACNF-2 spectra were recorded with spinning at 12 and $11 \mathrm{kHz}$, respectively. A $2.5 \mu \mathrm{s} 90^{\circ}$ proton pulse was followed by a variable contact time of 700 to $7500 \mu \mathrm{s}$, high power proton decoupling during acquisition, and a relaxation delay of $6 \mathrm{~s}$. We determined $T_{1 \rho \mathrm{H}}$ via CP experiments with varied ${ }^{1} \mathrm{H}$ spin-lock duration from 1,000 to $15,000 \mu$ s before application of the ${ }^{13} \mathrm{C}$ spin lock and a contact time of $2000 \mu \mathrm{s}$. For the $T_{1 \rho \mathrm{H}}$ experiments, phenyl ester CNF was spun at $9.5 \mathrm{kHz}$ and DACNF-2 was spun at $11 \mathrm{kHz}$. For all spectra, 1024 scans were acquired with a sweep width of 497 ppm. Each peak in the spectra was approximated by a Gaussian function curve fitting analysis.

Signal intensity $\left(M_{1}\right)$ was plotted as a function of contact time (t) for each ${ }^{13} \mathrm{C}$ species and the data were fit to the equation

$$
M_{1}=\frac{M_{0} \frac{\gamma_{H}}{\gamma_{C}}}{1-\frac{T_{C H}}{T_{1 \rho H}}}\left[e^{\left(-\frac{t}{T_{1 \rho H}}\right)}-e^{\left(-\frac{t}{T_{C H}}\right)}\right]
$$

where $\gamma_{\mathrm{H}}$ and $\gamma_{\mathrm{C}}$ are the gyromagnetic ratios of ${ }^{1} \mathrm{H}$ and ${ }^{13} \mathrm{C}, T_{\mathrm{CH}}$ is the cross-polarization time constant, and $M_{0}$ is the magnitude of the equilibrium magnetization which is directly proportional 
to the concentration of a given ${ }^{13} \mathrm{C}$ species in a sample. ${ }^{3}$ To fit the data from the set of standard $\mathrm{CP}$ experiments, calculated $T_{1 \rho \mathrm{H}}$ values were constrained while $M_{0}$ and $T_{\mathrm{CH}}$ values were varied through an iterative non-linear least squares fitting. The resulting curve fits are shown in Figure S11. We calculated $T_{1 \rho \mathrm{H}}$ from the negative inverse of the slope of a linear fit of $\ln$ (signal intensity) as a function of duration of ${ }^{1} \mathrm{H}$ spin-lock before contact time. For both samples, there appeared to be multiple $\mathrm{T}_{1 \rho \mathrm{H}}$ in the rotating frame affecting the signal intensities of the substituents, and a global $T_{1 \rho \mathrm{H}}$ affecting cellulose carbon signal intensity. Nonetheless, for phenyl ester CNF, the $T_{1 \rho \mathrm{H}}$ calculated for aromatic carbons $(3700 \pm 448.5 \mu$ s) allowed a good fit of the VCT data (Fig. S11). For DA-CNF-2, however, the $T_{1 \rho \mathrm{H}}$ calculated for the entire range of ${ }^{1} \mathrm{H}$ spin lock duration did not represent the observed decay in intensity in the VCT data. For this reason, the initial linear region $(1000-7000 \mu \mathrm{s})$ of the data was used to calculate a $T_{1 \rho \mathrm{H}}$ of $18000 \pm 1000 \mu \mathrm{s}$ which fit the VCT data well (Figure S11).

We calculated $\mathrm{DS}_{\text {overall }}$ for phenyl ester CNF according to the equation $\mathrm{DS}=1.2 \times M_{\mathrm{A}} / M_{\mathrm{C}}$, where $M_{\mathrm{A}}$ is the $M_{0}$ of aromatic carbons, $M_{\mathrm{C}}$ is the $M_{0}$ for cellulose carbons, and the factor of 1.2 normalizes the value to the number of carbons contributing to the intensity of each signal. We

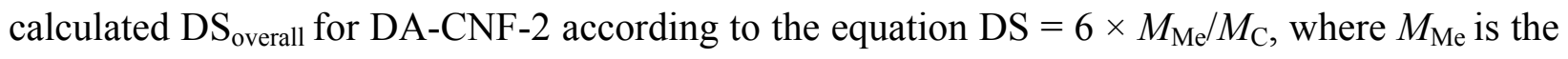
$M_{0}$ for the methyl carbon on the dodecyl ester substituent, and 6 is a normalization factor. The standard error in the $M_{0}$ values were propagated through the $\mathrm{DS}_{\text {overall }}$ calculations (Table S8).

The $\mathrm{DS}_{\text {overall }}$ for phenyl ester CNF was estimated to be 0.09 from the VCT experiments and was determined to be 0.14 by elemental analysis (Table S8). For the DA-CNF-2 sample, $\mathrm{DS}_{\text {overall }}$ estimated from VCT experiments was 0.19 while that obtained from elemental analysis was 0.45 . The $\mathrm{DS}_{\text {overall }}$ from VCT experiments is calculated from the normalized ratio of intensities of carbon on the substituent to the sum of intensities of cellulose carbons. We note that multiple $T_{1 \mathrm{pH}}$ 
behavior was observed for signals for the substituents on phenyl ester CNF and DA-CNF-2, which could be a source of error responsible for the disagreement between $\mathrm{DS}_{\text {overall }}$ from VCT and elemental analysis.

X-ray Photoelectron Spectroscopy. X-ray photoelectron spectra of CNF powders were obtained to determine $\mathrm{DS}_{\text {surface }}$ using a PHI 5600 XPS equipped with a Mg K $\alpha$ flood source (1253.6 eV) and a hemispherical energy analyzer. High resolution multiplex scans were collected at ultrahigh vacuum $\left(8 \times 10^{-8}\right.$ torr $)$ using a source power of $300 \mathrm{~W}$, pass energy $23.5 \mathrm{eV}, 10$ sweeps per spectrum, and $0.025 \mathrm{eV} /$ step. Survey scans were collected from $1200 \mathrm{eV}$ to $0 \mathrm{eV}$ at the same ultrahigh vacuum and power but with a pass energy of $187.85 \mathrm{eV}$, two sweeps per spectrum, and 1.6 eV/step. Spectra were analyzed using CASA XPS software.

\section{Biomethane Potential Tests}

Our assertion that the biogas measured as a comparison of biodegradation is supported by the comparatively low mass of carbon in the BMP media. The BMP media recipe shown in Table S3 is primarily inorganic, with trace amounts of organic nutrients. Each sample was digested in $100 \mathrm{~mL}$ of this media. By using the $\mathrm{wt} \%$ of $\mathrm{C}$ in each organic component of this media and diluting each stock to the final concentration in $100 \mathrm{~mL}$ of media (i.e., volume used to incubate samples), only $0.075 \mathrm{mg} \mathrm{C}$ is contributed by these nutrients. This is compared to $\sim 42 \mathrm{mg}$ of $\mathrm{C}$ added in a 100 mg sample of unmodified CNF. Importantly, these residual organics only contribute minimal biogas production as evidenced by our blank controls (media including sludge in absence of CNF sample) which produced $\sim 10 \mathrm{~mL}$ on average over 75 days compared to $>100 \mathrm{~mL}$ for CNF. The biogas evolved from the blanks primarily comes from the small organic content of the digested sludge. The overall carbon contribution from this component is small, as the $\%$ total solids (\%TS) of digested sludge is under $10 \%$, and often around 5-6\%, otherwise the anaerobic digester is not 
operating as intended, and samples would not have been available. ${ }^{4-6}$ For our tests, roughly $55 \%$ of the mass of these solids are considered "volatile" (data not shown). Included in these volatile solids (VS) are the biodegradable components of sludge, while the residual solids are typically inorganic or highly recalcitrant to biodegradation (i.e., they persist after heating at $550{ }^{\circ} \mathrm{C}$ for $2 \mathrm{hr}$ in standard sludge characterization tests). Because the volatile solids fraction is only partially biodegradable ( $\sim 60 \%$ for undigested sludge, less for digested sludge as was used here), each sample has only a small amount of theoretically biodegradable material contributed by the sludge, and only a portion of this material will consist of C. ${ }^{7}$ The minimal contribution of biogas by the blank controls suggests that most of the biogas produced in these BMP tests was evolved by the cellulosic samples being investigated.

\section{Aerobic Biodegradation Tests.}

Recovery of powdered samples was facilitated by the initial centrifugation step before the addition of primary effluent. By pelletizing each powder into the bottom of the vials prior to addition of the primary effluent, the solids were prevented from dispersing into the aqueous media. This permitted us to pipette off the supernatant media at the conclusion of the biodegradation experiment without disturbing the pelletized portion of the sample. Each powder was then recovered, washed with ethanol and MilliQ water, and dried before massing. While we cannot exclude a small contribution of residual biomass to the final mass of our samples, such a contribution can be safely neglected in comparing the relative biodegradability of the samples due to this washing step. 


\section{Tables}

Table S1. Elemental composition and calculated $\mathrm{DS}_{\text {overall }}$ for cellulose nanofibrils (CNFs) functionalized with ether and ester groups.

\begin{tabular}{|c|c|c|c|c|c|}
\hline Sample & \%C & \%H & \%N & \%O & DS $_{\text {overall }}$ \\
\hline Cellulose Nanofibrils & 41.53 & 6.07 & 0 & 52.4 & 0 \\
\hline Hexyl Ester CNF & 42.80 & 6.30 & 0 & 50.90 & 0.09 \\
\hline Phenyl Ester CNF & 44.75 & 5.86 & 0 & 49.39 & 0.14 \\
\hline Dodecyl Ether CNF & 45.41 & 6.85 & 0.11 & 47.63 & 0.11 \\
\hline Hexyl Ether CNF & 42.18 & 5.84 & 0.05 & 51.93 & 0.05 \\
\hline GP-HC-CNF-1 & - & - & - & - & - \\
\hline GP-HC-CNF-2 & - & - & - & - & - \\
\hline GP-HC-CNF-3 & - & - & - & - & - \\
\hline GP-HC-CNF-4 & 43.60 & 5.96 & 0.13 & 50.31 & 0.14 \\
\hline GP-LC-CNF-1 & - & - & - & - & - \\
\hline GP-LC-CNF-2 & - & - & - & - & - \\
\hline GP-LC-CNF-3 & - & - & - & - & - \\
\hline GP-LC-CNF-4 & 41.75 & 5.93 & 0.14 & 52.18 & 0.013 \\
\hline LC-CNF-1 & 58.45 & 9.31 & 0.55 & 31.69 & 0.80 \\
\hline LC-CNF-2 & 58.69 & 9.18 & 0.47 & 31.66 & 0.82 \\
\hline LC-CNF-3 & 55.26 & 8.51 & 0.36 & 35.87 & 0.56 \\
\hline LC-CNF-4 & 61.96 & 9.66 & 0.70 & 27.68 & 1.16 \\
\hline DA-CNF-1 & 42.56 & 6.27 & 0.08 & 51.09 & 0.035 \\
\hline DA-CNF-2 & 53.04 & 8.15 & 0.10 & 38.71 & 0.45 \\
\hline DA-CNF-3 & 49.47 & 7.42 & 0.08 & 43.03 & 0.28 \\
\hline DA-CNF-4 & 47.33 & 6.99 & 0.95 & 44.73 & 0.20 \\
\hline
\end{tabular}


Table S2. X-ray photoelectron spectra $\% \mathrm{C}-\mathrm{C}$ component values and calculated $\mathrm{DS}_{\text {surface }}$ for cellulose nanofibrils functionalized with different ether and ester groups.*

\begin{tabular}{|c|c|c|}
\hline Sample & \%C-C & $\mathrm{DS}_{\text {surface }}$ \\
\hline Cellulose Nanofibrils & 14.5 & 0 \\
\hline Hexyl Ester CNF & 17.1 & 0.03 \\
\hline Phenyl Ester CNF & 25.1 & 0.17 \\
\hline Dodecyl Ether CNF & 30.2 & 0.16 \\
\hline Hexyl Ether CNF & 25.2 & 0.25 \\
\hline GP-HC-CNF-1 & 53.1 & 1.19 \\
\hline GP-HC-CNF-2 & 58.7 & 1.54 \\
\hline GP-HC-CNF-3 & 63.2 & 1.89 \\
\hline GP-HC-CNF-4 & 68.4 & 2.43 \\
\hline GP-LC-CNF-1 & 20.7 & 0.07 \\
\hline GP-LC-CNF-2 & 30.8 & 0.17 \\
\hline GP-LC-CNF-3 & 35.8 & 0.23 \\
\hline GP-LC-CNF-4 & 42.7 & 0.33 \\
\hline LC-CNF-1 & 56.3 & 0.63 \\
\hline LC-CNF-2 & 57.8 & 0.67 \\
\hline LC-CNF-3 & 68.7 & 1.12 \\
\hline LC-CNF-4 & 82.3 & 2.46 \\
\hline DA-CNF-1 & 15.1 & 0.014 \\
\hline DA-CNF-2 & 24.2 & 0.097 \\
\hline DA-CNF-3 & 28.8 & 0.15 \\
\hline DA-CNF-4 & 47.9 & 0.43 \\
\hline
\end{tabular}


Table S3. Biomethane potential (BMP) test stock solutions S1-S7. BMP media consists of 0.9 $\mathrm{mL} \mathrm{S} 2,2.7 \mathrm{~mL} \mathrm{~S} 3,13.5 \mathrm{~mL} \mathrm{~S} 4,0.9 \mathrm{~mL} \mathrm{~S} 5,0.9 \mathrm{~mL} \mathrm{S6}$, and $9 \mathrm{~mL} \mathrm{~S} 7$ per liter of ultrapure water and $10 \%$ digested wastewater sludge.

\begin{tabular}{|c|c|c|}
\hline Solution & Compound & Concentration, $\mathrm{g} / \mathrm{L}$ \\
\hline S1 & Sample & $\begin{array}{l}<2 \mathrm{~g} / \mathrm{L} \text { degradable } \mathrm{COD} \text { in } \\
\text { assay liquid (estimated) }\end{array}$ \\
\hline $\mathrm{S} 2$ & Resazurin (oxidation-reduction indicator) & 1 \\
\hline S3 & $\left(\mathrm{NH}_{4}\right)_{2} \mathrm{HPO}_{4}$ & 26.7 \\
\hline \multirow[t]{10}{*}{ S4 } & $\mathrm{CaCl}_{2} \cdot 2 \mathrm{H}_{2} \mathrm{O}$ & 16.7 \\
\hline & $\mathrm{NH}_{4} \mathrm{CI}$ & 26.6 \\
\hline & $\mathrm{MgCl}_{2} \cdot 6 \mathrm{H}_{2} \mathrm{O}$ & 120 \\
\hline & $\mathrm{KCl}$ & 86.7 \\
\hline & $\mathrm{MnCl}_{2} \cdot 4 \mathrm{H}_{2} \mathrm{O}$ & 1.33 \\
\hline & $\mathrm{CoCl}_{2} \cdot 6 \mathrm{H}_{2} \mathrm{O}$ & 2 \\
\hline & $\mathrm{H}_{3} \mathrm{BO}_{3}$ & 0.38 \\
\hline & $\mathrm{CuCl}_{2} \cdot 2 \mathrm{H}_{2} \mathrm{O}$ & 0.18 \\
\hline & $\mathrm{Na}_{2} \mathrm{MoO}_{4} \cdot 2 \mathrm{H}_{2} \mathrm{O}$ & 0.17 \\
\hline & $\mathrm{ZnCl}_{2}$ & 0.14 \\
\hline S5 & $\mathrm{FeCl}_{2} \cdot 4 \mathrm{H}_{2} \mathrm{O}$ & 370 \\
\hline S6 & $\mathrm{Na}_{2} \mathrm{~S} \cdot 9 \mathrm{H}_{2} \mathrm{O}$ & 500 \\
\hline \multirow[t]{10}{*}{ S7 } & Biotin & 0.002 \\
\hline & Folic acid & 0.002 \\
\hline & Pyridoxine hydrochloride & 0.01 \\
\hline & Riboflavin & 0.005 \\
\hline & Thiamine & 0.005 \\
\hline & Nicotinic acid & 0.005 \\
\hline & Pantothenic acid & 0.005 \\
\hline & B12 & 0.0001 \\
\hline & p-aminobenzoic acid & 0.005 \\
\hline & Thioctic acid & 0.005 \\
\hline
\end{tabular}


Table S4. Calculated maximum biogas production from each functionalized cellulose nanofibril (CNF).

\begin{tabular}{|c|c|c|}
\hline Sample & $\begin{array}{c}\text { Calculated } \\
\text { Total Max } \\
\text { Biogas (mL/g) }\end{array}$ & $\begin{array}{c}\text { Empirical } \\
\text { Total Biogas } \\
\text { (mL/g) }\end{array}$ \\
\hline CNF & 680 & 680 \\
\hline PhAA CNF & 723 & 668 \\
\hline Hex Acid CNF & 674 & 694 \\
\hline Na $^{+}$TEMPO & 699 & 596 \\
\hline H $^{+}$TEMPO & 699 & 606 \\
\hline Ethyl Cellulose & 699 & 18 \\
\hline Hexyl Ether & 699 & 105 \\
\hline Dodecyl Ether & 699 & 70 \\
\hline DA-CNF-1 & 703 & 665 \\
\hline DA-CNF-2 & 883 & 828 \\
\hline DA-CNF-3 & 823 & 580 \\
\hline DA-CNF-4 & 791 & 524 \\
\hline LC-CNF-1 & 966 & 898 \\
\hline LC-CNF-2 & 970 & 687 \\
\hline LC-CNF-3 & 913 & 561 \\
\hline LC-CNF-4 & 1021 & 374 \\
\hline GP-HC-CNF-1 & 635 & 638 \\
\hline GP-HC-CNF-2 & 637 & 571 \\
\hline GP-HC-CNF-3 & 642 & 423 \\
\hline GP-HC-CNF-4 & 642 & 448 \\
\hline GP-LC-CNF-1 & 699 & 682 \\
\hline GP-LC-CNF-2 & 694 & 630 \\
\hline GP-LC-CNF-3 & 689 & 605 \\
\hline GP-LC-CNF-4 & 689 & 620 \\
\hline & & \\
\hline
\end{tabular}


Table S5. Gompertz model statistics and parameters* for functionalized cellulose nanofibrils (CNFs).

\begin{tabular}{|c|c|c|c|c|c|c|}
\hline Sample & RMSE & NRMSE & $\mathbf{R}^{2}$ & Normalized $\boldsymbol{V}_{\max }$ & Normalized $\boldsymbol{K}$ & Normalized $\boldsymbol{I}$ \\
\hline CNF & 0.058 & 0.250 & 0.977 & 1.01 & 0.078 & 0.68 \\
\hline LC-CNF-1 & 0.026 & 0.080 & 0.995 & 0.93 & 0.036 & 3.01 \\
\hline LC-CNF-2 & 0.030 & 0.086 & 0.989 & 0.71 & 0.031 & 2.82 \\
\hline LC-CNF-3 & 0.033 & 0.181 & 0.976 & 0.62 & 0.017 & 0.00 \\
\hline LC-CNF-4 & 0.022 & 0.151 & 0.979 & 0.37 & 0.019 & 2.96 \\
\hline DA-CNF-1 & 0.052 & 0.138 & 0.986 & 0.95 & 0.037 & 2.70 \\
\hline DA-CNF-2 & 0.095 & 0.289 & 0.937 & 0.94 & 0.020 & 0.00 \\
\hline DA-CNF-3 & 0.092 & 0.312 & 0.897 & 0.70 & 0.012 & 0.00 \\
\hline DA-CNF-4 & 0.048 & 0.251 & 0.968 & 0.67 & 0.0090 & 0.00 \\
\hline GP-HC-CNF-1 & 0.024 & 0.172 & 0.996 & 1.01 & 0.031 & 5.19 \\
\hline GP-HC-CNF-2 & 0.022 & 0.127 & 0.995 & 0.90 & 0.023 & 3.20 \\
\hline GP-HC-CNF-3 & 0.022 & 0.079 & 0.990 & 0.66 & 0.013 & 0.00 \\
\hline GP-HC-CNF-4 & 0.025 & 0.123 & 0.988 & 0.70 & 0.013 & 0.49 \\
\hline GP-LC-CNF-1 & 0.045 & 0.410 & 0.987 & 0.98 & 0.082 & 1.57 \\
\hline GP-LC-CNF-2 & 0.043 & 0.163 & 0.986 & 0.91 & 0.046 & 2.43 \\
\hline GP-LC-CNF-3 & 0.052 & 0.191 & 0.975 & 0.88 & 0.029 & 0.00 \\
\hline GP-LC-CNF-4 & 0.040 & 0.201 & 0.987 & 0.90 & 0.036 & 3.39 \\
\hline Hexyl Ester CNF & 0.056 & 0.236 & 0.986 & 1.03 & 0.095 & 2.32 \\
\hline Phenyl Ester CNF & 0.018 & 0.202 & 0.996 & 0.93 & 0.011 & 10.79 \\
\hline Na Carboxyl CNF & 0.018 & 0.043 & 0.997 & 0.85 & 0.016 & 2.42 \\
\hline Ethyl Cellulose & 0.005 & 1.486 & 0.636 & 0.03 & 0.00 & 0.58 \\
\hline H $^{+}$Carboxyl CNF & 0.062 & 0.224 & 0.964 & 0.87 & 0.0031 & 0.00 \\
\hline Hexyl Ether CNF & 0.021 & 2.047 & 0.865 & 0.15 & 0.73 \\
\hline Dodecyl Ether CNF & 0.020 & 0.494 & 0.664 & 0.10 & 0.00 \\
\hline
\end{tabular}

* Parameters include root mean squared error (RMSE), normalized root mean square error (NRMSE), coefficient of determination $\left(\mathrm{R}^{2}\right)$, maximum normalized biogas production $\left(V_{\max }\right)$, biogas production rate $(K)$, and lag phase $(I)$. 
Table S6. Assignment of ${ }^{13} \mathrm{C}-\mathrm{NMR}$ chemical shifts $\left(\delta_{13 \mathrm{C}}\right)$ for untreated cellulose nanofibrils $(\mathrm{CNF})$ and functionalized nanocellulose samples.*

\begin{tabular}{|c|c|c|c|c|c|c|c|c|c|c|}
\hline \multicolumn{11}{|c|}{$\delta_{13 \mathrm{c}}(\mathrm{ppm})$} \\
\hline & $C=0$ & Aromatic C & C1 & C4 (C) & C4 (A) & $C 2,3,5$ (C) & $C 2,3,5(A)$ & C6 (C) & C6 (A) & Aliphatic C \\
\hline CNF & $\mathrm{n} / \mathrm{a}$ & $\mathrm{n} / \mathrm{a}$ & $\begin{array}{c}105.4 \\
102.5^{+}\end{array}$ & 89.3 & $\begin{array}{l}83.5 \\
82.3^{+}\end{array}$ & $\begin{array}{l}73.0 \\
72.0^{+}\end{array}$ & $\begin{array}{l}75.3 \\
74.6^{\dagger}\end{array}$ & 65.5 & $\begin{array}{r}63.0 \\
61.5^{+}\end{array}$ & $\mathrm{n} / \mathrm{a}$ \\
\hline Phenyl Ester CNF & $\begin{array}{c}172.7 \\
174.7^{+}\end{array}$ & $\begin{array}{l}134.5 \\
129.5\end{array}$ & $\begin{array}{c}105.3 \\
101.9^{+}\end{array}$ & 89.1 & $\begin{array}{l}83.0 \\
84.1^{+}\end{array}$ & $\begin{array}{l}72.6 \\
71.9\end{array}$ & $\begin{array}{l}75.2 \\
74.6^{+}\end{array}$ & 65.4 & $\begin{array}{r}63.4 \\
62.8^{+}\end{array}$ & $41.3,42.2^{+}$ \\
\hline Hexyl Ester CNF & 176.0 & $n / a$ & $\begin{array}{l}104.7 \\
97.7^{+}\end{array}$ & 88.9 & $\begin{array}{l}82.2 \\
83.6^{\dagger}\end{array}$ & 72.9 & 75.1 & 65.2 & $\begin{array}{r}62.5 \\
60.0^{+}\end{array}$ & $34.6,31.7,25.9,22.9,14.4$ \\
\hline $\mathrm{Na}^{+}$Carboxyl CNF & $\begin{array}{r}175.7 \\
177.6^{+}\end{array}$ & $n / a$ & 105.3 & $\begin{array}{l}89.0 \\
87.5^{+}\end{array}$ & $\begin{array}{l}83.8 \\
81.4^{+}\end{array}$ & $\begin{array}{r}72.7 \\
71.6^{+} \\
\end{array}$ & $\begin{array}{r}74.9 \\
74.5^{+} \\
\end{array}$ & 65.2 & $\begin{array}{r}63.1 \\
59.0^{+} \\
\end{array}$ & $\mathrm{n} / \mathrm{a}$ \\
\hline Hexyl Ether CNF & $\mathrm{n} / \mathrm{a}$ & $\mathrm{n} / \mathrm{a}$ & $\begin{array}{c}104.2 \\
100.5^{+}\end{array}$ & 88.6 & 82.7 & 72.1 & 75.0 & 65.4 & 62.7 & $32.8,31.0,30.4,27.5^{\dagger}, 26.8,23.6,15.3$ \\
\hline DA-CNF-2 & 181.9 & $n / a$ & 105.2 & 89.0 & $\begin{array}{l}83.7 \\
81.4^{+}\end{array}$ & 72.0 & 75.1 & 65.5 & $\begin{array}{l}63.3 \\
60.8^{+}\end{array}$ & $\begin{array}{c}35.5^{\dagger}, 35.0,33.1,32.0^{\dagger}, 30.5,25.0 \\
23.4^{+}, 22.3^{+}, 16.5^{\dagger}, 15.1\end{array}$ \\
\hline DA-CNF-3 & 181.6 & $\mathrm{n} / \mathrm{a}$ & 105.2 & 88.7 & 83.1 & 72.0 & 74.7 & 65.2 & 62.4 & $34.7^{\dagger}, 32.7,30.0,24.6,14.7$ \\
\hline DA-CNF-4 & 181.2 & $\mathrm{n} / \mathrm{a}$ & $\begin{array}{l}104.6 \\
100.8^{+}\end{array}$ & 88.6 & $\begin{array}{l}83.4 \\
82.0\end{array}$ & $\begin{array}{l}71.2 \\
72.1^{+}\end{array}$ & $\begin{array}{l}74.7 \\
73.7^{+}\end{array}$ & $\begin{array}{l}64.6 \\
65.3^{+}\end{array}$ & $\begin{array}{r}62.5 \\
61.2^{+} \\
59.6^{+} \\
\end{array}$ & $\begin{array}{c}34.1,33.2^{\dagger}, 32.4,31.3^{\dagger}, 29.9,25.4^{\dagger} \\
24.3,22.7^{\dagger}, 14.4,12.2,10.6\end{array}$ \\
\hline LC-CNF-1 & 181.0 & $\mathrm{n} / \mathrm{a}$ & $\begin{array}{c}104.4 \\
101.2^{+}\end{array}$ & 88.3 & $\begin{array}{l}83.1 \\
80.0^{+}\end{array}$ & $\begin{array}{l}71.7 \\
71.2\end{array}$ & $\begin{array}{r}74.3 \\
73.6^{\dagger}\end{array}$ & $\begin{array}{r}64.6 \\
65.4^{+}\end{array}$ & $\begin{array}{r}62.1 \\
61.3^{+}\end{array}$ & $41.5,34.2,32.3,31.4^{\dagger}, 29.6,24.2,14.3$ \\
\hline GP-LC-CNF-4 & $\mathrm{n} / \mathrm{a}$ & $\mathrm{n} / \mathrm{a}$ & $\begin{array}{c}105.1 \\
102.4^{+}\end{array}$ & 89.0 & $\begin{array}{l}83.6 \\
82.4^{+}\end{array}$ & $\begin{array}{r}72.7 \\
71.9^{\dagger}\end{array}$ & $\begin{array}{r}75.1 \\
74.5^{\dagger}\end{array}$ & $\begin{array}{r}65.2 \\
66.0^{+}\end{array}$ & $\begin{array}{r}62.7 \\
60.9^{+}\end{array}$ & $44.5,43.7,26.3^{\dagger}, 23.2,21.8,20.5^{\dagger}$ \\
\hline
\end{tabular}

* Crystalline (C) and amorphous (A) forms are designated.

$\dagger$ Indicates a shoulder. 
Table S7. Proportion of carbon 1-6 that is crystalline or amorphous in cellulose nanofibrils (CNFs) functionalized with the indicated ether, ester, and carboxyl groups.*

\begin{tabular}{|c|c|c|c|c|c|c|c|c|c|c|}
\hline \multicolumn{10}{|c|}{ Sample } & \multicolumn{10}{c|}{${ }^{13}$ C-4rystallinity } & \multicolumn{2}{c|}{ C-2,3,5 } & \multicolumn{2}{c|}{ C-6 } & \multicolumn{2}{c|}{ Average \%C } & \multicolumn{2}{c|}{ Average \%A } \\
\cline { 2 - 13 } & \%C & \%A & \%C & \%A & \%C & \%A & Avg & St Dev & Avg & St Dev \\
\hline CNF & 38 & 62 & 39 & 61 & 40 & 60 & 39 & 1.0 & 61 & 1.0 \\
\hline Phenyl Ester CNF & 48 & 52 & 48 & 52 & 49 & 51 & 49 & 0.6 & 52 & 0.8 \\
\hline Hexyl Ester CNF & 31 & 69 & 32 & 68 & 35 & 65 & 33 & 2.1 & 67 & 2.1 \\
\hline Na $^{+}$Carboxyl CNF & 39 & 61 & 41 & 59 & 40 & 60 & 40 & 1.0 & 60 & 1.0 \\
\hline Hexyl Ether CNF & 43 & 57 & 44 & 56 & 45 & 55 & 44 & 1.2 & 56 & 1.2 \\
\hline LC-CNF-1 & 51 & 49 & 50 & 50 & 51 & 49 & 51 & 1.0 & 49 & 1.0 \\
\hline DA-CNF-2 & 58 & 42 & 56 & 44 & 60 & 40 & 58 & 1.5 & 42 & 1.5 \\
\hline DA-CNF-3 & 56 & 44 & 55 & 45 & 57 & 43 & 56 & 1.1 & 44 & 1.1 \\
\hline DA-CNF-4 & 50 & 50 & 51 & 49 & 52 & 48 & 51 & 0.9 & 49 & 0.9 \\
\hline GP-LC-CNF-4 & 43 & 57 & 42 & 58 & 43 & 57 & 43 & 0.8 & 57 & 0.8 \\
\hline
\end{tabular}

* Crystalline (C), Amorphous (A), Cellulose Nanofibrils (CNF), Dodecanoic Acid (DA), and Gas Phase Lauroyl Chloride (GP-LC) are designated

Table S8. Computed $T_{1 \mathrm{pH}}, T_{\mathrm{CH}}, M_{0}$, and DS values for carbon species in phenyl ester CNF and DACNF-2

\begin{tabular}{|l|l|c|c|c|c|}
\hline \multicolumn{1}{|c|}{ Sample } & Carbon Type & $\boldsymbol{T}_{\mathbf{1 \rho H}}(\boldsymbol{\mu s})$ & $\boldsymbol{T}_{\mathrm{CH}}(\boldsymbol{\mu s})$ & $\boldsymbol{M}_{\mathbf{0}}$ & DS \\
\hline \multirow{2}{*}{ Phenyl ester Cellulose } & Aromatic & $3700 \pm 450$ & $200 \pm 140$ & $4500 \pm 160$ & $0.087 \pm 0.003$ \\
\cline { 2 - 5 } & Cellulose & $6400 \pm 150$ & $180 \pm 77$ & $62400 \pm 910$ & \\
\hline \multirow{2}{*}{ DA-CNF-2 } & Methyl & $18000 \pm 1000$ & $390 \pm 21$ & $1170 \pm 8$ & $0.192 \pm 0.001$ \\
\cline { 2 - 5 } & Cellulose & $9600 \pm 380$ & $247 \pm 5$ & $36600 \pm 52$ & \\
\hline
\end{tabular}


Table S9. $\mathrm{DS}_{\text {surface }}$ and $\mathrm{DS}_{\text {overall }}$ values and Gompertz fit parameters for unmodified and functionalized cellulose nanofibrils.*

\begin{tabular}{|c|c|c|c|c|}
\hline Sample & $\mathrm{DS}_{\text {surface }}$ & $\mathrm{DS}_{\text {overall }}$ & Normalized $K$ & Normalized $V_{\max }$ \\
\hline \multicolumn{5}{|c|}{ Unmodified Cellulose Nanofibrils } \\
\hline Cellulose Nanofibrils & 0 & 0 & 0.078 & 1.01 \\
\hline \multicolumn{5}{|c|}{ Liquid Phase Dodecanoic Acid Functionalization } \\
\hline DA-CNF-1 & 0.014 & 0.035 & 0.037 & 0.95 \\
\hline DA-CNF-2 (Dodecyl Ester CNF) & 0.097 & 0.45 & 0.020 & 0.94 \\
\hline DA-CNF-3 & 0.15 & 0.28 & 0.012 & 0.70 \\
\hline DA-CNF-4 & 0.43 & 0.20 & 0.009 & 0.67 \\
\hline \multicolumn{5}{|c|}{ Liquid Phase Lauroyl Chloride Functionalization } \\
\hline LC-CNF-1 & 0.63 & 0.80 & 0.036 & 0.93 \\
\hline LC-CNF-2 & 0.67 & 0.82 & 0.031 & 0.71 \\
\hline LC-CNF-3 & 1.12 & 0.56 & 0.017 & 0.62 \\
\hline LC-CNF-4 & 2.46 & 1.16 & 0.019 & 0.37 \\
\hline \multicolumn{5}{|c|}{ Gas Phase Hexanoyl Chloride Functionalization } \\
\hline GP-HC-CNF-1 & 1.19 & 0.17 & 0.031 & 1.01 \\
\hline GP-HC-CNF-2 & 1.54 & 0.16 & 0.023 & 0.90 \\
\hline GP-HC-CNF-3 & 1.89 & 0.14 & 0.013 & 0.66 \\
\hline GP-HC-CNF-4 & 2.43 & 0.14 & 0.013 & 0.70 \\
\hline \multicolumn{5}{|c|}{ Gas Phase Lauroyl Chloride Functionalization } \\
\hline GP-LC-CNF-1 & 0.066 & 0.008 & 0.082 & 0.98 \\
\hline GP-LC-CNF-2 & 0.17 & 0.015 & 0.046 & 0.91 \\
\hline GP-LC-CNF-3 & 0.23 & 0.012 & 0.029 & 0.88 \\
\hline GP-LC-CNF-4 & 0.33 & 0.013 & 0.036 & 0.90 \\
\hline
\end{tabular}

* Normalized rate $(K)$ and maximum biogas production (Vmax) were obtained from Gompertz fits of normalized biogas production data. Cellulose Nanofibrils (CNF), Dodecanoic Acid (DA), Lauroyl Chloride (LC), Gas Phase Hexanoyl Chloride (GP-HC) and Gas Phase Lauroyl Chloride (GP-LC) are denoted. 


\section{Figures}

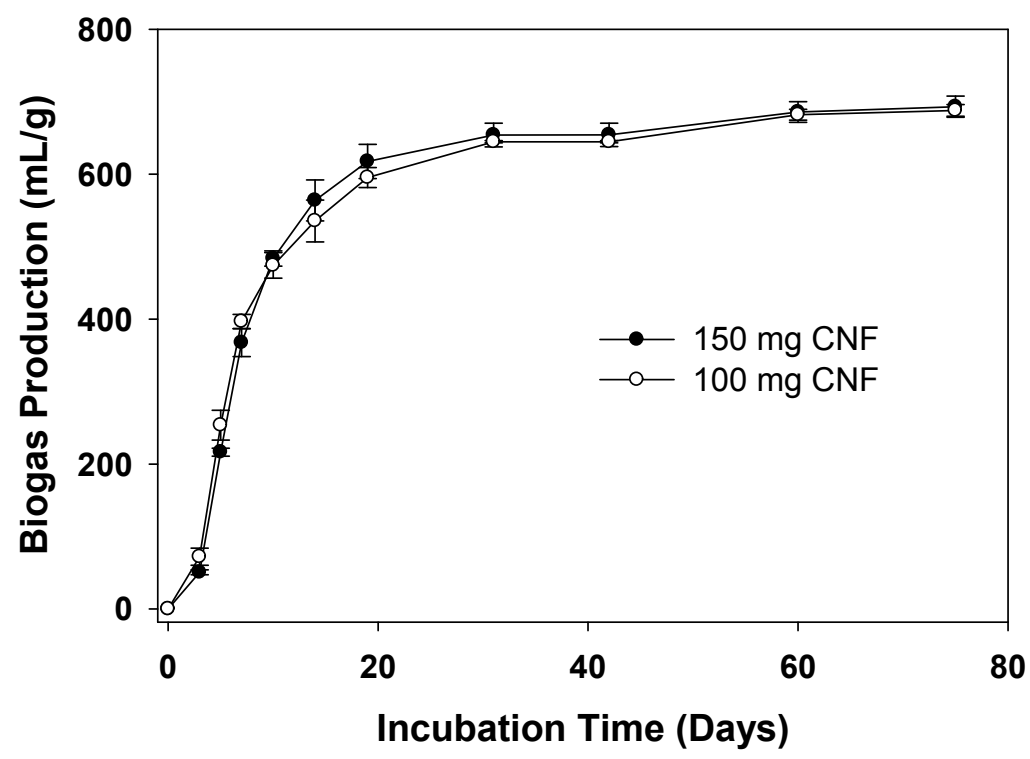

Figure S1. Biogas production during mineralization of $150 \mathrm{mg}$ (black circles) and $100 \mathrm{mg}$ (white circles) cellulose nanofibrils (CNF) by an anaerobic microbial community. Biogas production was normalized for mass loading. Error bars represent one standard deviation from triplicate samples.
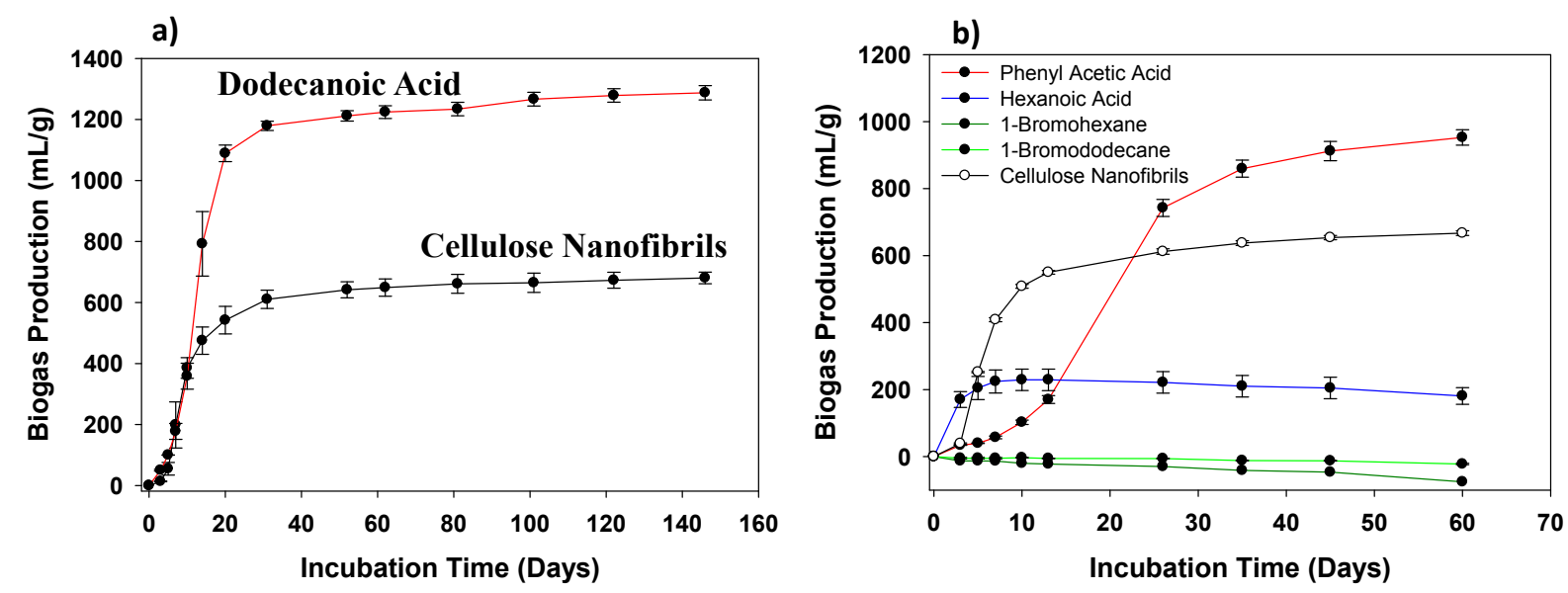

Figure S2. a) Biogas production during mineralization of dodecanoic acid and cellulose nanofibrils by an anaerobic microbial community. b) Biogas production during mineralization of functionalization reagents. In both panels, error bars represent one standard deviation from the average of duplicate samples. 


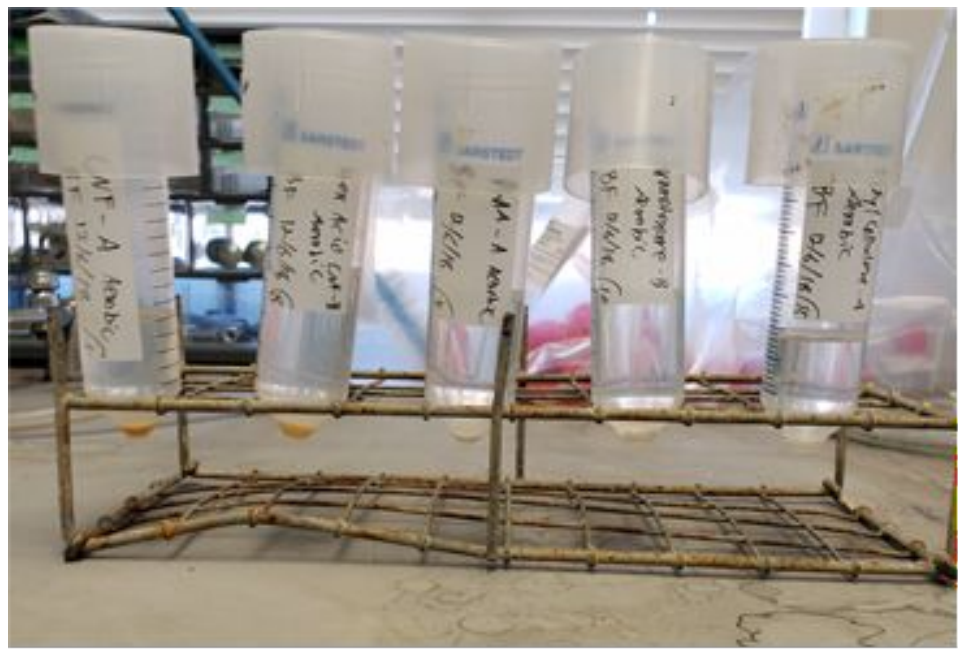

Figure S3. Digital images of functionalized cellulose nanofibrils after $60 \mathrm{~d}$ of exposure to an aerobic microbial community in $50 \mathrm{~mL}$ conical vials.

a)

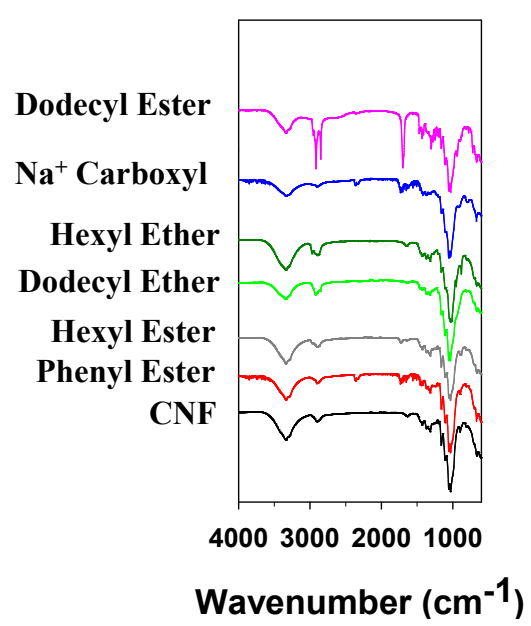

b)

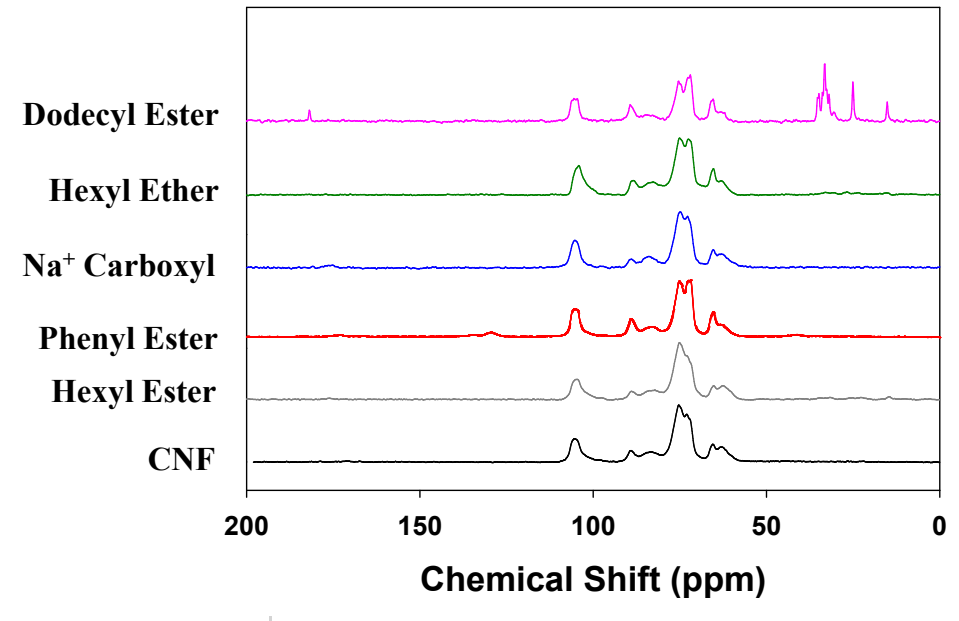

Figure S4. a) Infrared and b) ${ }^{13} \mathrm{C}$-NMR spectra of cellulose nanofibrils (black) functionalized with dodecyl (pink), hexyl (grey), and phenyl (red) esters as well as hexyl (dark green) and dodecyl (light green) ethers and carboxylic acid (blue). 
a)

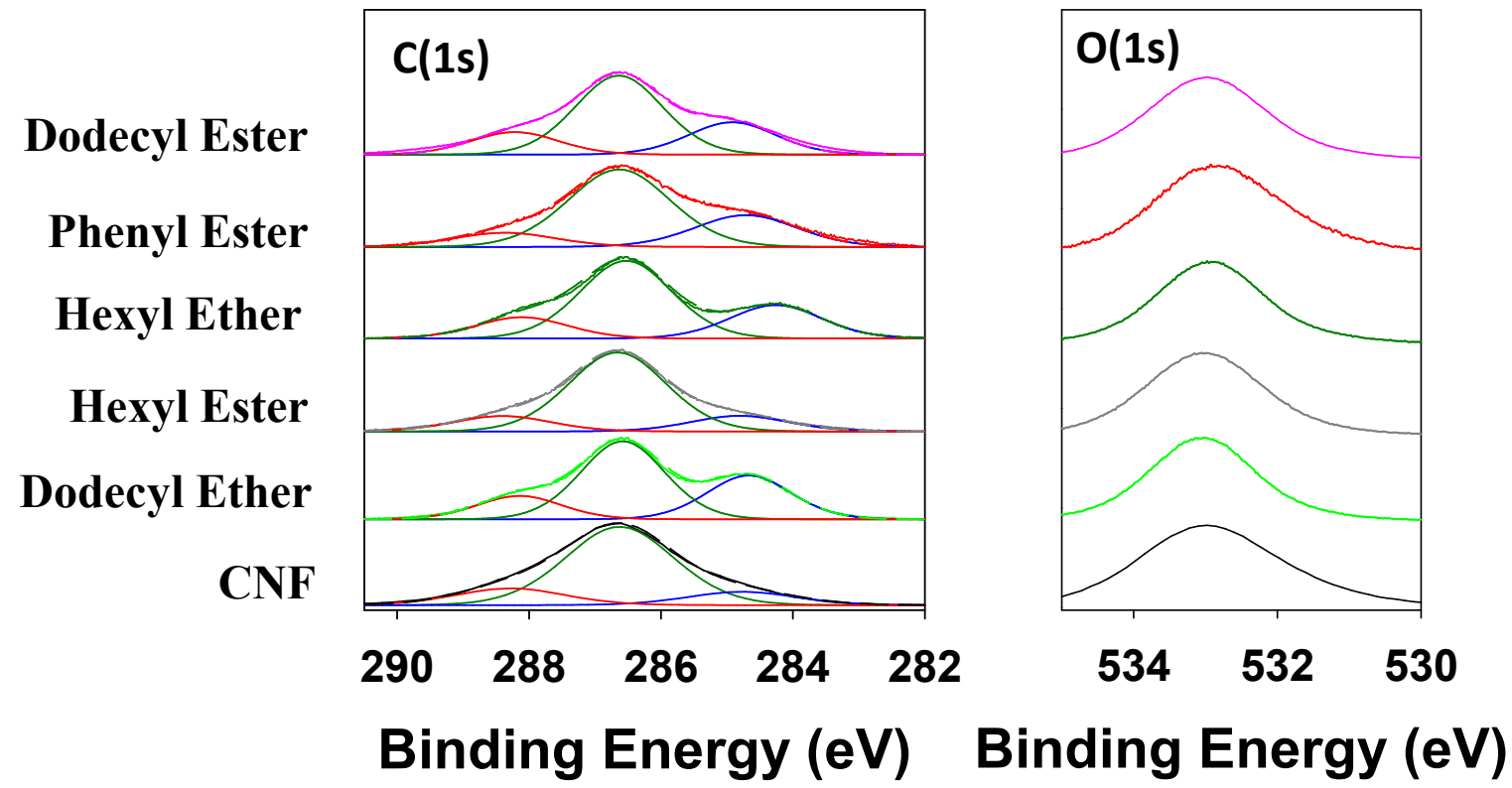

Figure S5. X-ray photoelectron spectra. a) C(1s) with component peak fitting and b) O(1s) regions of cellulose nanofibrils (CNFs) and CNFs functionalized with dodecyl (pink), phenyl (red), and hexyl (grey) esters and dodecyl (light green) and hexyl (dark green) ethers.

a)

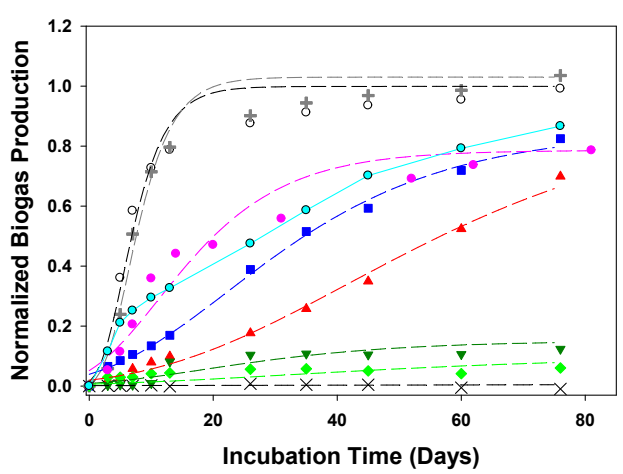

b)

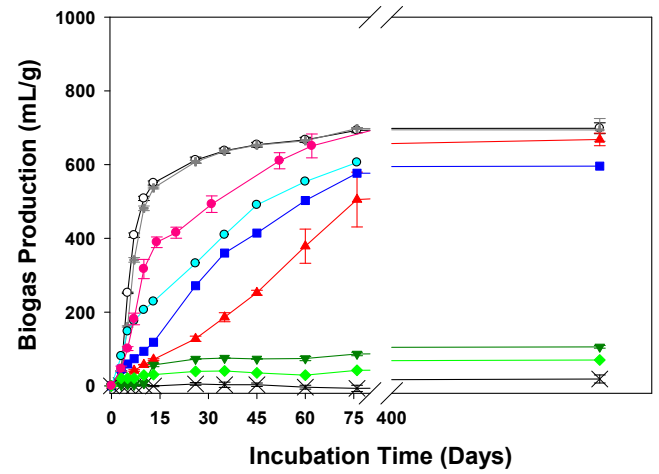

Figure S6. a) Normalized (dotted lines are fits from Gompertz model) and b) unnormalized biogas production (not fitted, as denoted by solid lines) from the mineralization of cellulose nanofibrils (CNF) and CNFs functionalized with hexyl ester, dodecyl ester, carboxylic acid with $\mathrm{Na}^{+}$and $\mathrm{H}^{+}$counterions, phenyl ester, hexyl ether, and dodecyl ether as well as commercial ethyl macrocellulose. Error bars represent one standard deviation of duplicate samples. 


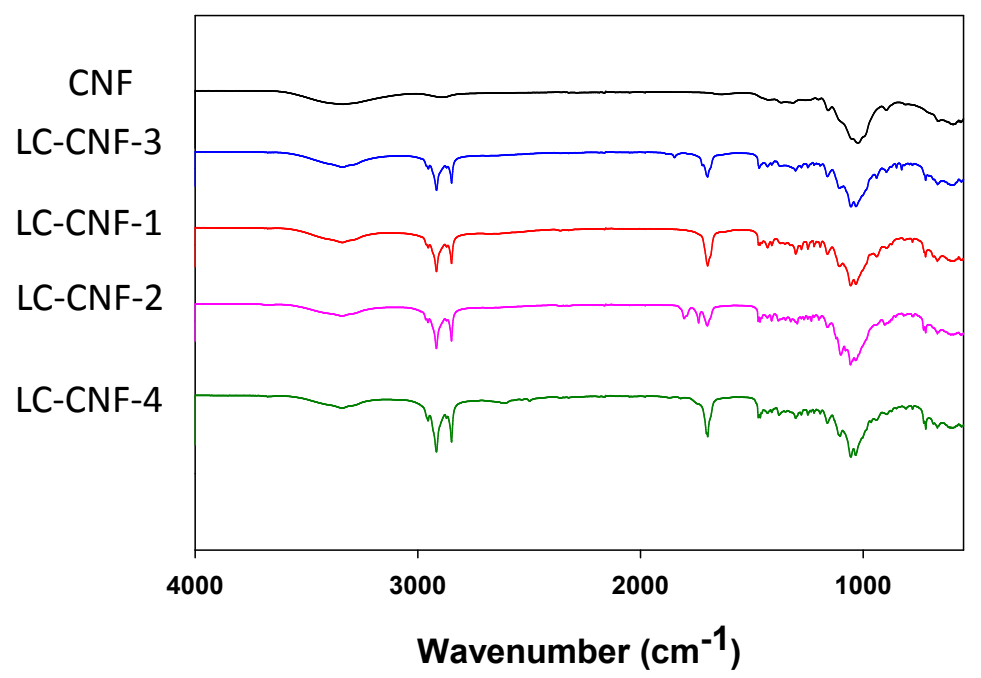

Figure S7. Infrared spectra of cellulose nanofibrils (CNF) esterified with lauroyl chloride (LC-CNF).

a)

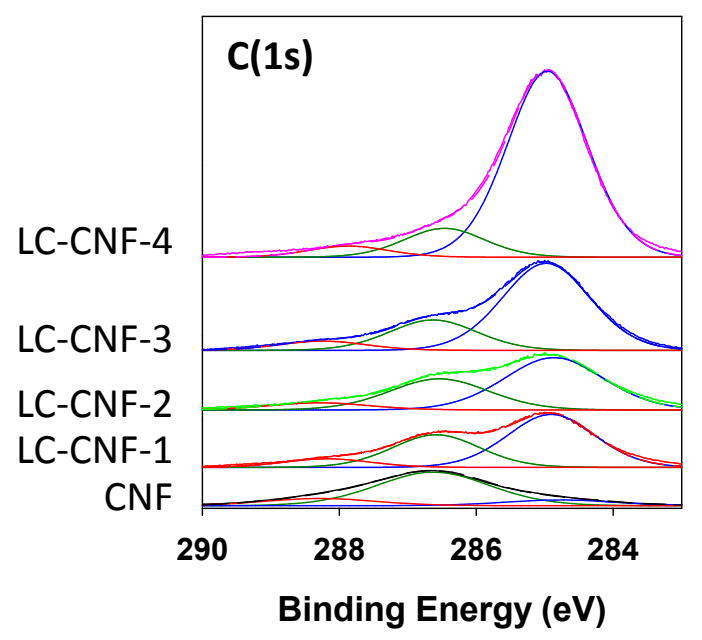

b)

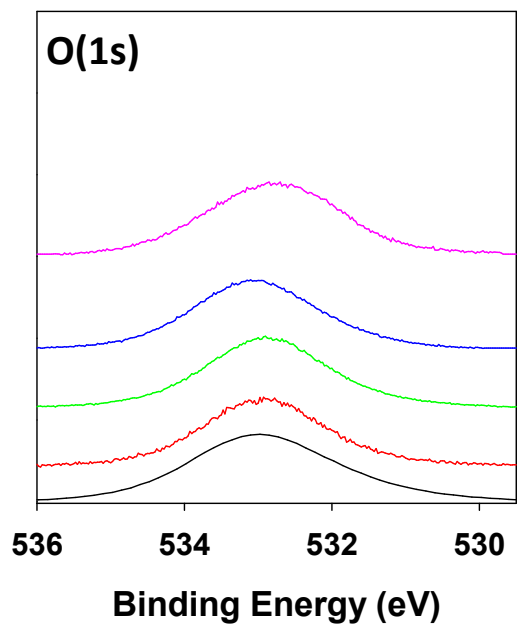

Figure S8. X-ray photoelectron spectra. a) Fitted C(1s) and b) $\mathrm{O}(1 \mathrm{~s})$ regions of cellulose nanofibrils (CNF) esterified with lauroyl chloride (LC-CNF). 


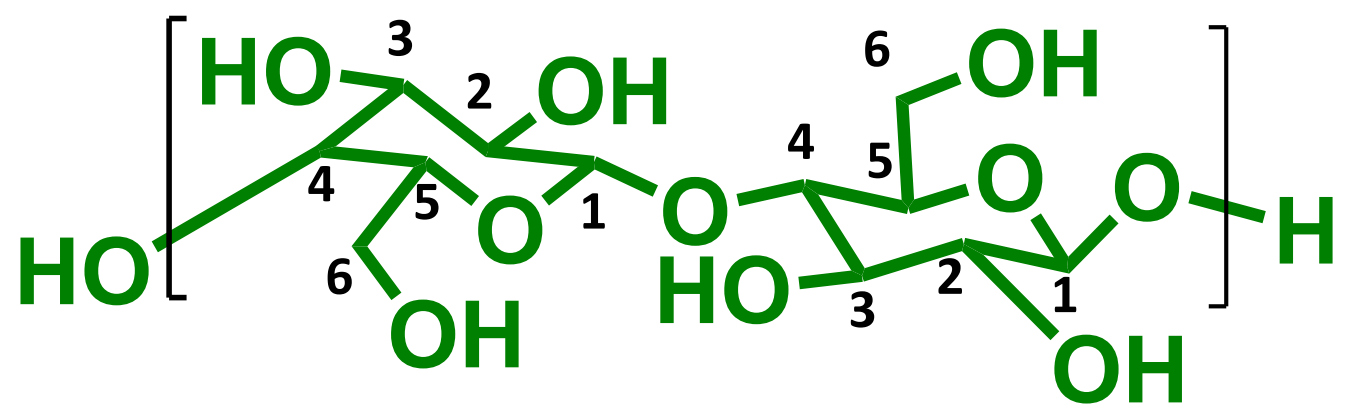

Figure S9. Nanocellulose repeat unit (cellobiose) with carbons 1-6 labeled for each glucose subunit.

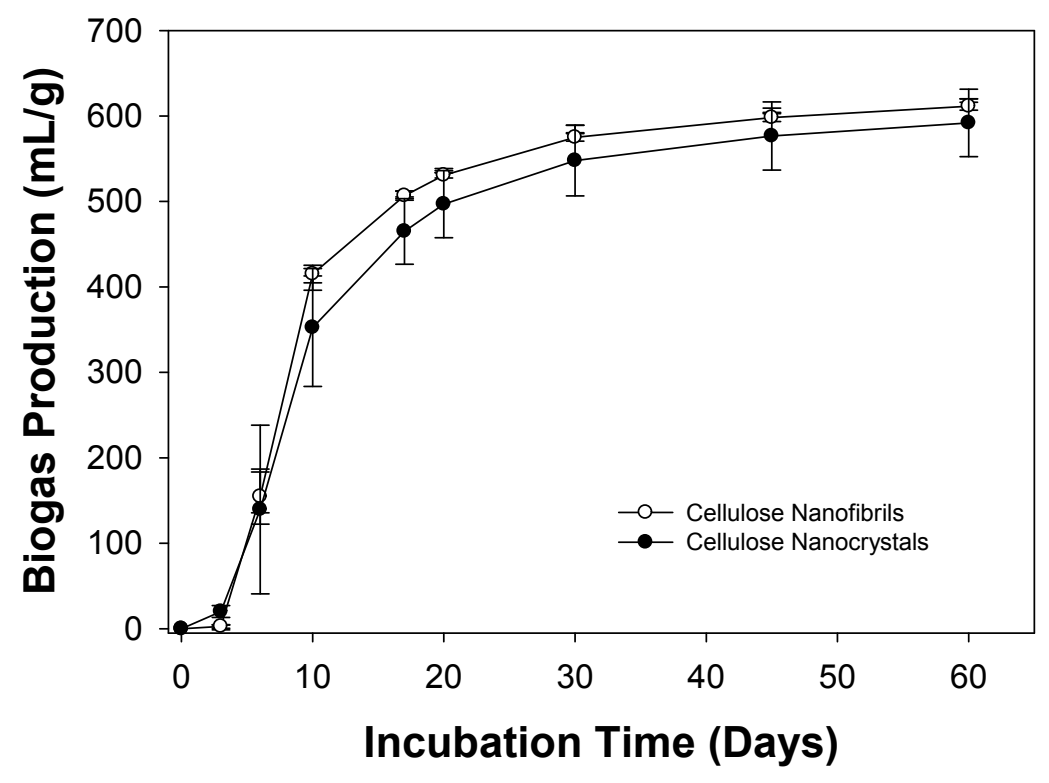

Figure S10. Biogas production of cellulose nanofibrils (CNFs) compared to cellulose nanocrystals (CNCs). Data is not fit to the Gompertz model as denoted by the solid connecting lines. Error bars represent one standard deviation from triplicate samples. 
a)

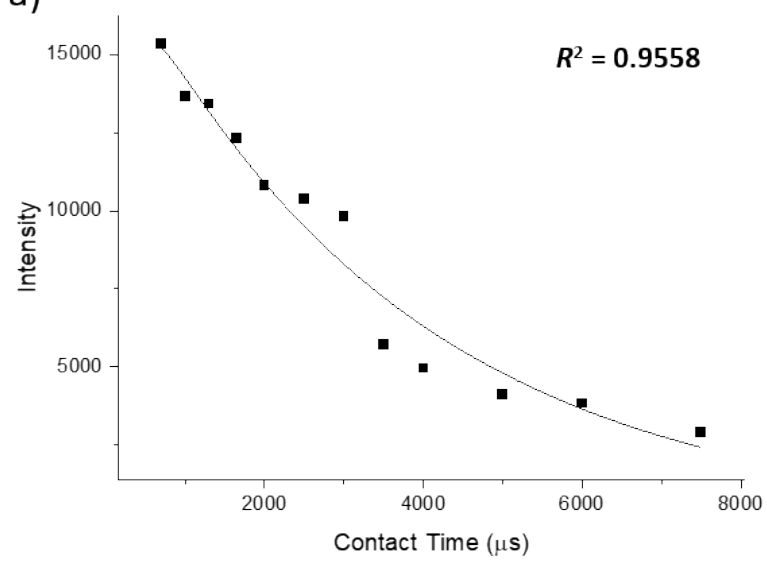

c)

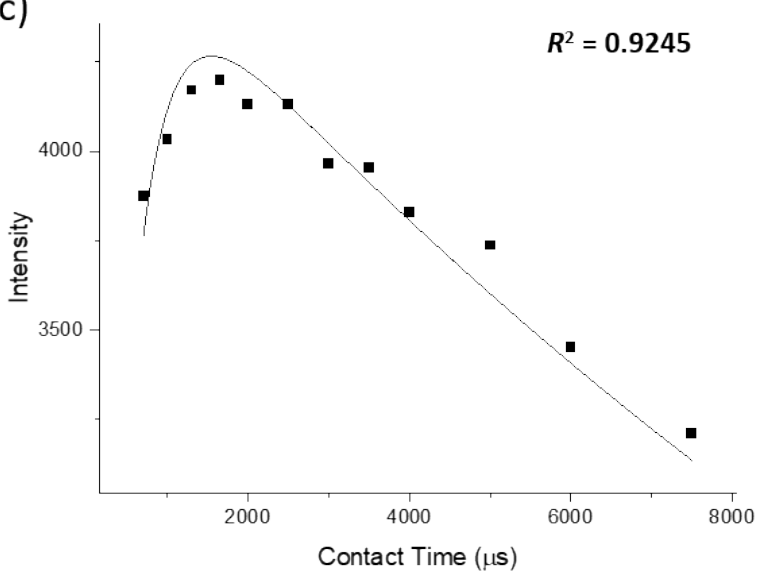

b)

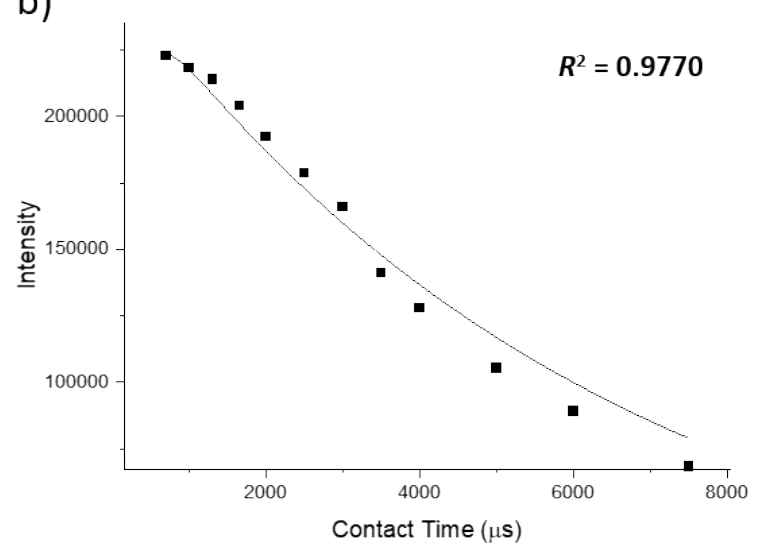

d)

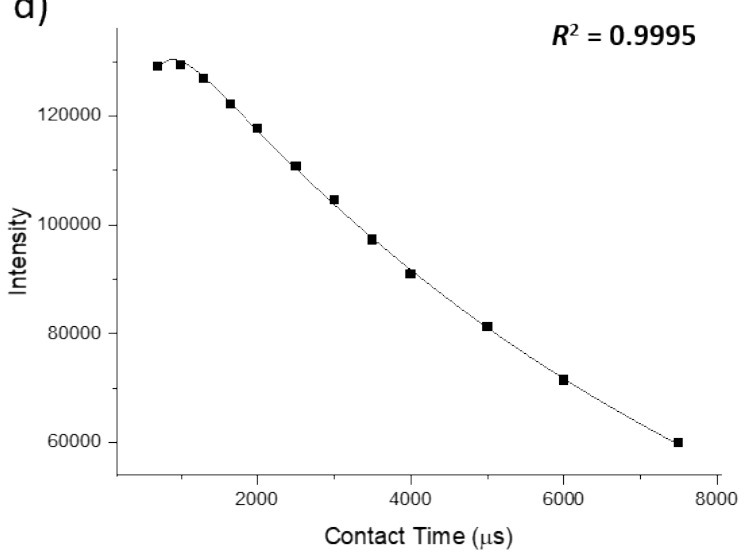

Figure S11. Signal intensity as a function of contact time for a) phenyl ester CNF aromatic carbons, b) phenyl ester CNF cellulose carbons, c) DA-CNF-2 methyl carbons, and d) DACNF-2 cellulose carbons from variable contact time CP-MAS experiments. Symbols represent experimental data. Lines correspond to least squares fit according to equation $\mathrm{S} 1$ with $R^{2}$ values displayed. 
a)

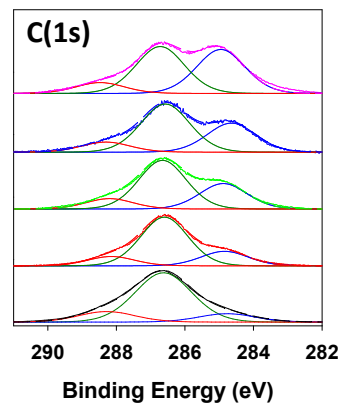

Lauroyl Chloride

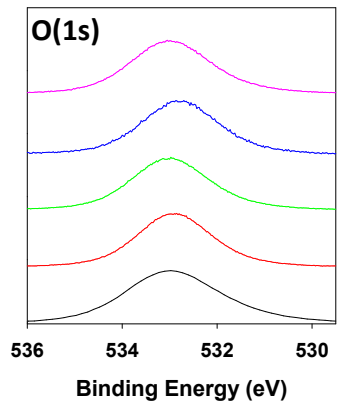

b)

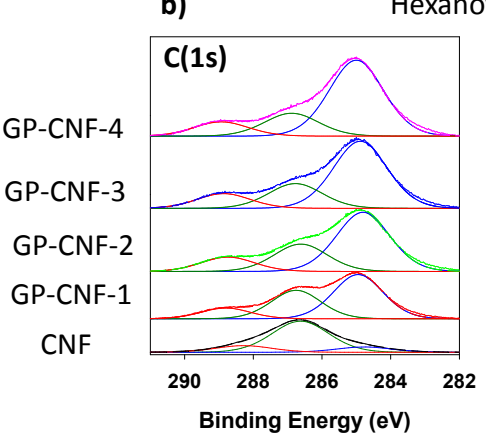

Hexanoyl Chloride

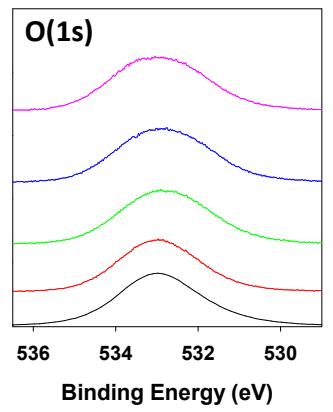

Figure S12. Fits of $X$-ray photoelectron spectra for the $\mathrm{C}(1 \mathrm{~s})$ and $\mathrm{O}(1 \mathrm{~s})$ regions for cellulose nanofibrils (CNFs) functionalized with gas phase (GP-CNF) a) lauroyl chloride and b) hexanoyl chloride.

a)

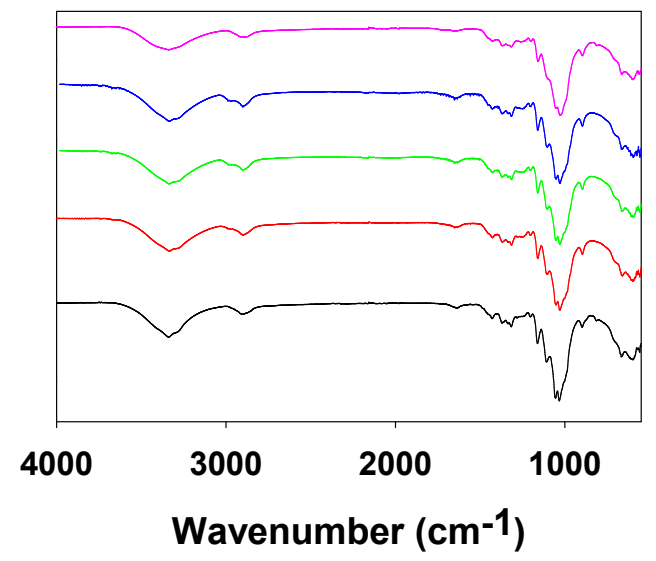

b)

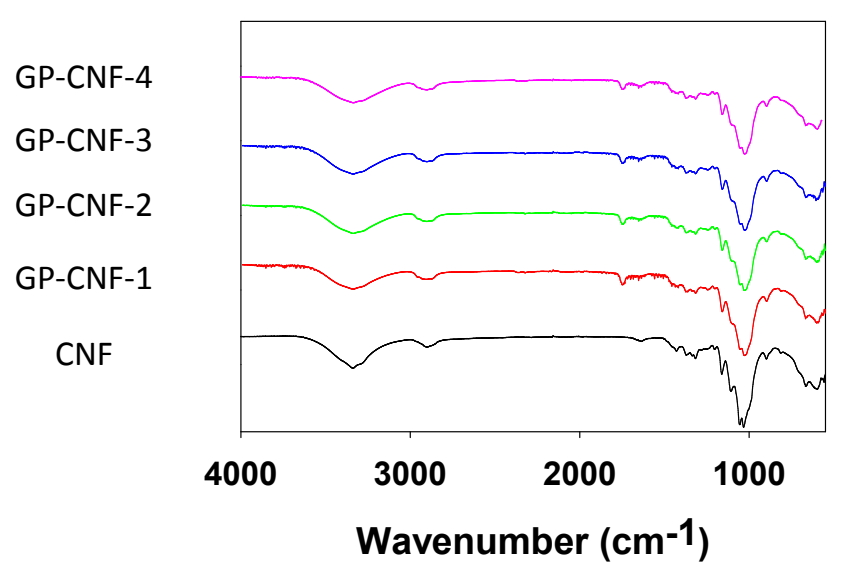

Figure S13. Infrared spectra of cellulose nanofibrils (CNF) esterified with gas phase (GP) a) lauroyl chloride (LC-CNF) and b) hexanoyl chloride (HC-CNF). 
a)

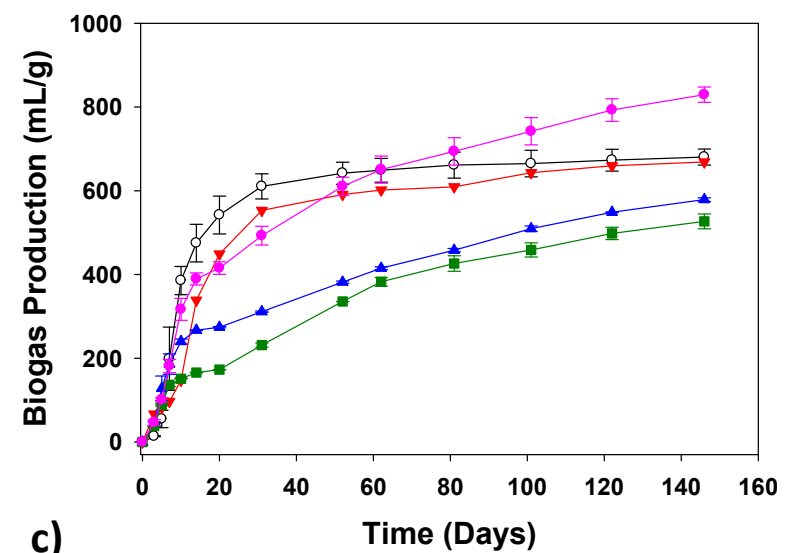

c)

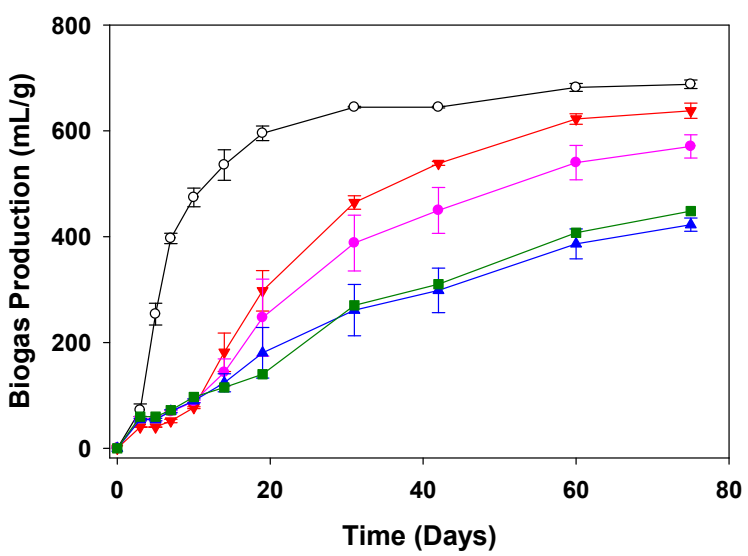

b)
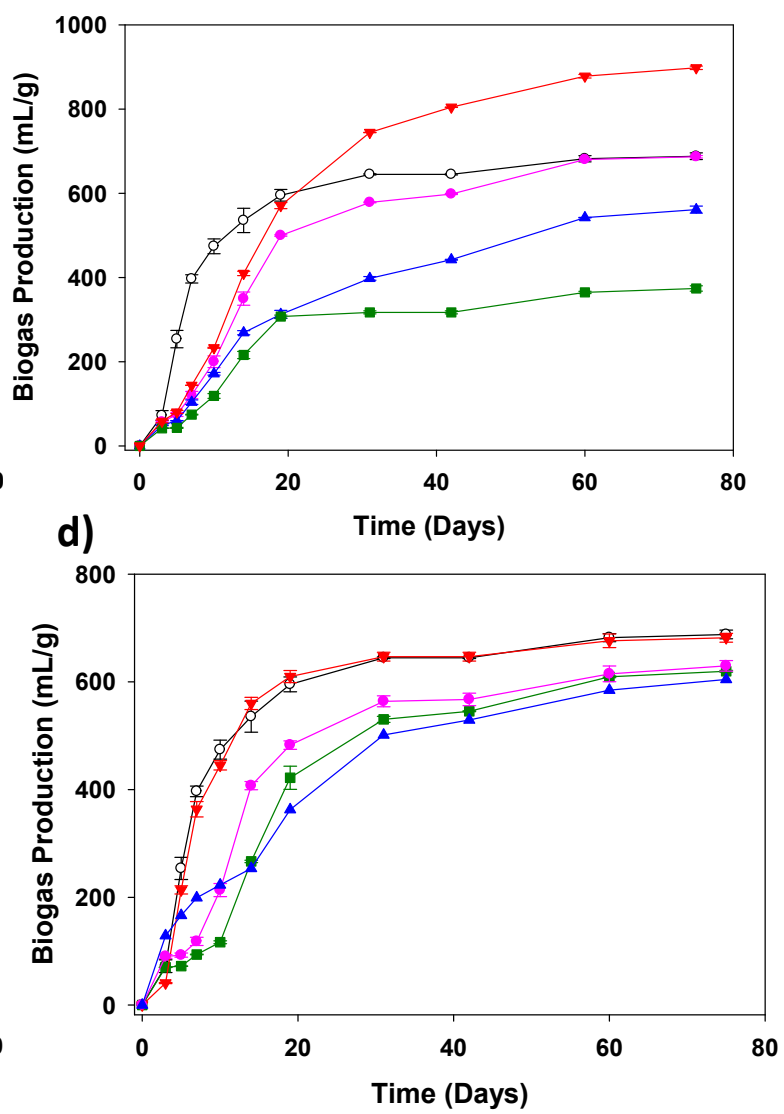

Figure S14. Unnormalized biogas production of cellulose nanofibrils functionalized with a) liquidphase dodecanoic acid, b) liquid-phase lauroyl chloride, c) gas-phase hexanoyl chloride, and d) gasphase lauroyl chloride during mineralization by an anaerobic microbial community. In each sample set, the number corresponding to degree of surface substitution increases from 1-4 as: red, pink, blue, green. In each plot, error bars represent one standard deviation of the average from duplicate samples. 
a)

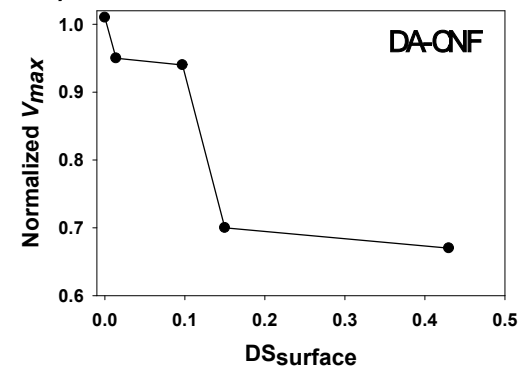

b)

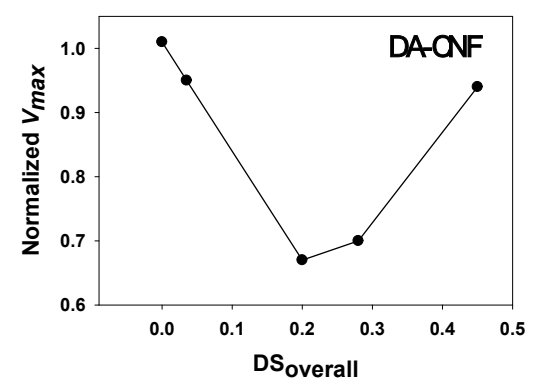

c)

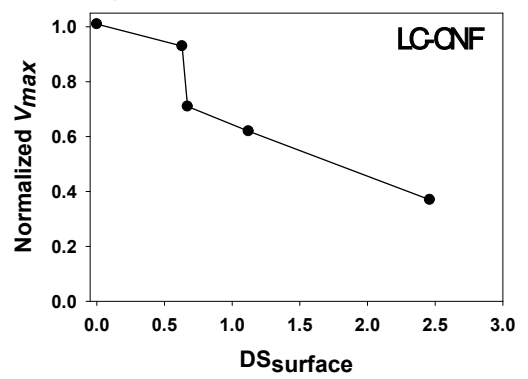

d)

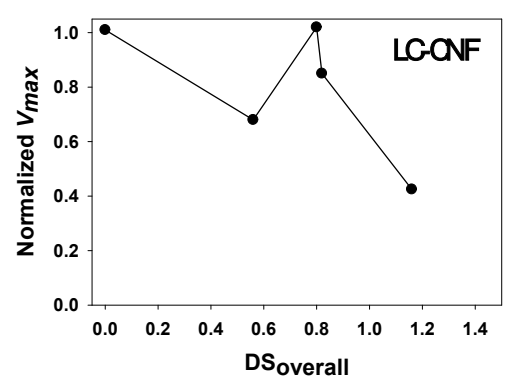

e)
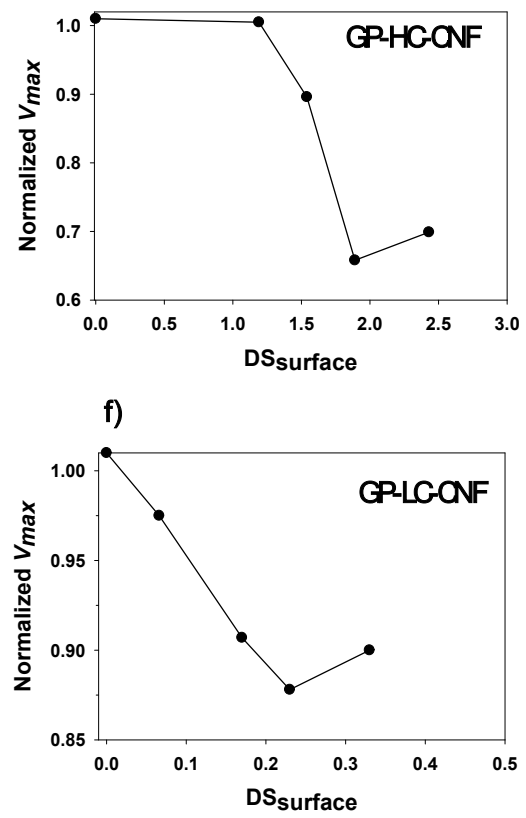

Figure S15. Relation of $\mathrm{DS}_{\text {surface }}$ and $\mathrm{DS}_{\text {overall }}$ with normalized maximum biogas production $\left(V_{\max }\right)$ for CNF esters functionalized with (a,b) dodecanoic acid (DA) and (c,d) lauroyl chloride (LC). Also shown is the correlation of $\mathrm{DS}_{\text {surface }}$ with normalized maximum biogas production for CNF esters functionalized with (e) gas phase (GP) hexanoyl chloride (HC) and (f) gas phase lauroyl chloride. 


\section{REFERENCES}

1. Frank, B. P.; Durkin, D. P.; Caudill, E. R.; Zhu, L.; White, D. H.; Curry, M. L.; Pedersen, J. A.; Fairbrother, D. H., Impact of Silanization on the Structure, Dispersion Properties, and Biodegradability of Nanocellulose as a Nanocomposite Filler. ACS Applied Nano Materials 2018, 1 (12), 7025-7038.

2. Loof, D.; Hiller, M.; Oschkinat, H.; Koschek, K., Quantitative and Qualitative Analysis of Surface Modified Cellulose Utilizing TGA-MS. Materials 2016, 9 (6), 415.

3. Kinchesh, P.; Powlson, D. S.; Randall, E. W., C-13 Nmr-Studies of Organic-Matter in Whole Soils .1. Quantitation Possibilities. Eur J Soil Sci 1995, 46 (1), 125-137.

4. Dai, X.; Chen, Y.; Zhang, D.; Yi, J., High-solid Anaerobic Co-digestion of Sewage Sludge and Cattle Manure: The Effects of Volatile Solid Ratio and pH. Sci. Rep. 2016, 6 (1), 35194.

5. Mei, R.; Narihiro, T.; Nobu, M. K.; Kuroda, K.; Liu, W.-T., Evaluating digestion efficiency in fullscale anaerobic digesters by identifying active microbial populations through the lens of microbial activity. Sci. Rep. 2016, 6 (1), 34090.

6. Yi, J.; Dong, B.; Jin, J.; Dai, X., Effect of increasing total solids contents on anaerobic digestion of food waste under mesophilic conditions: performance and microbial characteristics analysis. PloS one 2014, 9 (7), e102548-e102548.

7. Baptista, M. Modelling of the kinetics of municipal solid waste composting in full-scale mechanical biological treatment plants. New University of Lisbon, 2009. 